SUPPLEMENTARY INFORMATION

\title{
Efficient Catalysis of Nazarov Cyclization Using a Cationic Iridium Complex Possessing Adjacent Labile Coordination Sites
}

\author{
Mesfin Janka, Wei He, Alison J. Frontier* and Richard Eisenberg* \\ Department of Chemistry, University of Rochester, Rochester, NY 14627
}

\section{Experimental Section}

General Procedures and Materials. Chloroform- $\mathrm{d}$ and dichloromethane- $\mathrm{d}_{2}$ were purchased from Cambridge Isotope. [IrMe(CO)(dppe)(DIB)](BARF) $2,{ }^{1,2} \mathrm{NaBARF},{ }^{3}$ N-crotonyl-2-oxazolidinone ${ }^{4}$ and all of the Nazarov substrates ${ }^{5}$ were synthesized according to published procedures. The cyclized products were characterized as reported before. ${ }^{5}$

Column chromatography was performed on Silica Gel (40-140 mesh). TLC visualization was accomplished using UV light or with $\mathrm{KMnO}_{4}$ solution. Dichloromethane, diethylether, hexanes, pentane, and tetrahydrofuran were purified as described by Grubbs. ${ }^{6}$ All NMR spectra were recorded on a Bruker AMX or Avance $400 \mathrm{MHz}$ spectrometer. ${ }^{1} \mathrm{H}$ and ${ }^{13} \mathrm{C}$ chemical shifts ( $\square$ in ppm) are relative to tetramethylsilane and referenced using chemical shifts of residual solvent resonances. ${ }^{31} \mathrm{P}$ chemical shifts $(\square$ in ppm) are relative to an external $85 \%$ solution of phosphoric acid in the appropriate solvent.

Unless otherwise stated, all reactions and manipulations were performed in dry glassware under a nitrogen atmosphere using either standard Schlenk techniques or an inertatmosphere glovebox. 
Binding study of Nazarov substrate 2-(benzo[1,3]dioxole-5-carbonyl)-3-(2,4,6trimethoxy-phenyl)-acrylic acid methyl ester and $\left[\operatorname{IrMe}(\mathrm{CO})(\mathrm{dppe})(\mathrm{DIB})_{2}\right][\mathrm{BARF}]_{2}$. In a glovebox, an NMR tube was charged with 2-(benzo[1,3]dioxole-5-carbonyl)-3(2,4,6-trimethoxy-phenyl)-acrylic acid methyl ester (50.0 mg, $0.12 \mathrm{mmol})$ and $[\mathrm{IrMe}(\mathrm{CO})(\mathrm{dppe})(\mathrm{DIB})][\mathrm{BARF}]_{2}(6.48 \mathrm{mg}, 0.0024 \mathrm{mmol})$ and sealed with septum. The tube was cooled to $-78{ }^{\circ} \mathrm{C}, 0.60 \mathrm{~mL}$ of $\mathrm{CD}_{2} \mathrm{Cl}_{2}$ added and quickly transferred to precooled NMR spectrometer. It was then warmed to $-10{ }^{\circ} \mathrm{C}$ and ${ }^{31} \mathrm{P}\left\{{ }^{1} \mathrm{H}\right\}$ NMR spectrum recorded. ${ }^{31} \mathrm{P}\left\{{ }^{1} \mathrm{H}\right\} \mathrm{NMR}\left(\mathrm{CD}_{2} \mathrm{Cl}_{2}\right)$ at $-10{ }^{\circ} \mathrm{C}$ for the major isomer $(55 \%)$ : $\square 30.5\left(\mathrm{~d}, J_{\mathrm{P}-\mathrm{P}}\right.$ $=4.8 \mathrm{~Hz}, 1 \mathrm{P}$, trans to $\mathrm{CO}), 15.8\left(\mathrm{~d}, J_{\mathrm{P}-\mathrm{P}}=4.8 \mathrm{~Hz}, 1 \mathrm{P}\right.$, cis to $\left.\mathrm{CO}\right)$. For the minor isomer (45\%): $\square 30.0\left(\mathrm{~d}, J_{\mathrm{P}-\mathrm{P}}=4.8 \mathrm{~Hz}, 1 \mathrm{P}\right.$, trans to $\left.\mathrm{CO}\right), 17.3\left(\mathrm{~d}, J_{\mathrm{P}-\mathrm{P}}=4.8 \mathrm{~Hz}, 1 \mathrm{P}\right.$, cis to $\left.\mathrm{CO}\right)$. Binding study of Nazarov substrate 1-cyclohex-1-enyl-3-(2,4,6-trimethoxy-phenyl)propenone and $\left[\operatorname{IrMe}(\mathrm{CO})(\mathrm{dppe})(\mathrm{DIB})_{2}\right][\mathrm{BARF}]_{2}$. The same procedure was followed as described above for binding study of Nazarov substrate 2-(benzo[1,3]dioxole-5carbonyl)-3-(2,4,6-trimethoxy-phenyl)-acrylic acid methyl ester and $\left[\mathrm{IrMe}(\mathrm{CO})(\mathrm{dppe})(\mathrm{DIB})_{2}\right][\mathrm{BARF}]_{2}$. 1-Cyclohex-1-enyl-3-(2,4,6-trimethoxy-phenyl)propenone (36.0 mg, $0.12 \mathrm{mmol}),\left[\mathrm{IrMe}(\mathrm{CO})(\mathrm{dppe})(\mathrm{DIB})_{2}\right][\mathrm{BARF}]_{2}(6.48 \mathrm{mg}, 0.0024$ mmol), and $0.60 \mathrm{~mL} \mathrm{CD} \mathrm{Cl}_{2}$ were used. ${ }^{31} \mathrm{P}\left\{{ }^{1} \mathrm{H}\right\} \operatorname{NMR}\left(\mathrm{CD}_{2} \mathrm{Cl}_{2}\right)$ at $-10{ }^{\circ} \mathrm{C}$ for the major isomer (87\%): $\square 24.1\left(\mathrm{~d}, J_{\mathrm{P}-\mathrm{P}}=6.0 \mathrm{~Hz}, 1 \mathrm{P}\right.$, trans to $\left.\mathrm{CO}\right), 13.8\left(\mathrm{~d}, J_{\mathrm{P}-\mathrm{P}}=6.0 \mathrm{~Hz}\right.$, 1P, cis to CO). For the minor isomer (13\%): $\square 26.3\left(\mathrm{~d}, J_{\mathrm{P}-\mathrm{P}}=6.0 \mathrm{~Hz}, 1 \mathrm{P}\right.$, trans to $\left.\mathrm{CO}\right)$, $18.4\left(\mathrm{~d}, J_{\mathrm{P}-\mathrm{P}}=6.0 \mathrm{~Hz}, 1 \mathrm{P}\right.$, cis to $\left.\mathrm{CO}\right)$.

Binding study of Nazarov substrate 1-(5,6-dihydro-4H-pyran-2-yl)-3-(2,4,6trimethoxy-phenyl)-propenone and $\left[\operatorname{IrMe}(\mathrm{CO})(\mathrm{dppe})(\mathrm{DIB})_{2}\right][\mathrm{BARF}]_{2}$. The same 
procedure was followed as described above for the binding study of Nazarov substrate 2(benzo[1,3]dioxole-5-carbonyl)-3-(2,4,6-trimethoxy-phenyl)-acrylic acid methyl ester and $\left[\operatorname{IrMe}(\mathrm{CO})(\mathrm{dppe})(\mathrm{DIB})_{2}\right][\mathrm{BARF}]_{2} \cdot$ 1-(5,6-Dihydro-4H-pyran-2-yl)-3-(2,4,6-

trimethoxy-phenyl)-propenone (37.0 mg, $0.12 \mathrm{mmol})$, $\left[\mathrm{IrMe}(\mathrm{CO})(\mathrm{dppe})(\mathrm{DIB})_{2}\right][\mathrm{BARF}]_{2}$ (6.48 $\mathrm{mg}, 0.0024 \mathrm{mmol}$ ), and $0.60 \mathrm{~mL} \mathrm{CD}_{2} \mathrm{Cl}_{2}$ were used. The ${ }^{31} \mathrm{P}$ NMR spectrum $\left(\mathrm{CD}_{2} \mathrm{Cl}_{2}\right)$ was recorded at $-20{ }^{\circ} \mathrm{C} .{ }^{31} \mathrm{P}\left\{{ }^{1} \mathrm{H}\right\} \mathrm{NMR}\left(\mathrm{CD}_{2} \mathrm{Cl}_{2}\right)$ at $-20{ }^{\circ} \mathrm{C}$ for the major isomer (67\%): $\square 31.7\left(\mathrm{~d}, J_{\mathrm{P}-\mathrm{P}}=4.8 \mathrm{~Hz}, 1 \mathrm{P}\right.$, trans to $\left.\mathrm{CO}\right), 16.0\left(\mathrm{~d}, J_{\mathrm{P}-\mathrm{P}}=6.0 \mathrm{~Hz}, 1 \mathrm{P}\right.$, cis to CO). For the minor isomer (33\%): $\square 32.8\left(\mathrm{~d}, J_{\mathrm{P}-\mathrm{P}}=5.1 \mathrm{~Hz}, 1 \mathrm{P}\right.$, trans to CO), $16.0(\mathrm{~d}$, $J_{\mathrm{P}-\mathrm{P}}=5.1 \mathrm{~Hz}, 1 \mathrm{P}$, cis to CO).

\section{Binding study of N-crotonyl-2-oxazolidinone and $\left[\operatorname{IrMe}(\mathrm{CO})(\mathrm{dppe})(\mathrm{DIB})_{2}\right][\mathrm{BARF}]_{2}$.}

In a glovebox, an NMR tube was charged with N-crotonyl-2-oxazolidinone (1.0 mg, $0.0063 \mathrm{mmol}),[\mathrm{IrMe}(\mathrm{CO})(\mathrm{dppe})(\mathrm{DIB})][\mathrm{BARF}]_{2}(17.0 \mathrm{mg}, 0.0063 \mathrm{mmol})$ and $0.60 \mathrm{~mL}$ of $\mathrm{CD}_{2} \mathrm{Cl}_{2}$ and sealed with septum. NMR spectral analysis at $25^{\circ} \mathrm{C}$ demonstrated conversion to the new products and free 1,2-diiodobenzene. Spectral information for the major isomer (66\%): ${ }^{1} \mathrm{H} N M R\left(\mathrm{CD}_{2} \mathrm{Cl}_{2}\right)$ at $25{ }^{\circ} \mathrm{C}: \quad \square 6.86-8.0$ (overlapping, phenyl, BARF, olefinic), $6.04\left(\mathrm{dt}, J_{\mathrm{H}-\mathrm{H}}=14.5\right.$ and $1.6 \mathrm{~Hz}, 1 \mathrm{H}$, olefinic), 2.50-5.00 (overlapping, $\mathrm{PCH}_{2} \mathrm{CH}_{2} \mathrm{P}$, oxazolidinone), 2.21 (dd, $J_{\mathrm{H}-\mathrm{H}}=7.3$ and $1.4 \mathrm{~Hz}$, $\left.3 \mathrm{H}, \mathrm{CH}_{3} \mathrm{CHCHCO}\right), 0.97\left(\mathrm{dd}, J_{\mathrm{P}-\mathrm{H}}=5.7\right.$ and $\left.2.0 \mathrm{~Hz}, 3 \mathrm{H}, \mathrm{Ir}-\mathrm{CH}_{3}\right) .{ }^{31} \mathrm{P}\left\{{ }^{1} \mathrm{H}\right\} \mathrm{NMR}$ $\left(\mathrm{CD}_{2} \mathrm{Cl}_{2}\right)$ at $25^{\circ} \mathrm{C}: \mathrm{\square} 32.7\left(\mathrm{~d}, J_{\mathrm{P}-\mathrm{P}}=4.7 \mathrm{~Hz}, J_{\mathrm{P}-\mathrm{C}}=130 \mathrm{~Hz}, 1 \mathrm{P}\right.$, trans to $\left.\mathrm{CO}\right), 15.1\left(\mathrm{~d}, J_{\mathrm{P}-\mathrm{P}}\right.$ $=4.7 \mathrm{~Hz}, 1 \mathrm{P}$, cis to $\mathrm{CO})$. Spectral information for the minor isomer $(34 \%):{ }^{1} \mathrm{H} \mathrm{NMR}$ $\left(\mathrm{CD}_{2} \mathrm{Cl}_{2}\right)$ at $25^{\circ} \mathrm{C}: \quad \square 6.86-8.0$ (overlapping, phenyl, $\mathrm{BARF}$, olefinic), $5.76\left(\mathrm{dq}, J_{\mathrm{H}-\mathrm{H}}=\right.$ 14.5 and $1.8 \mathrm{~Hz}, 1 \mathrm{H}$, olefinic), 2.50-5.00 (overlapping, $\mathrm{PCH}_{2} \mathrm{CH}_{2} \mathrm{P}$, oxazolidinone), 2.01 
$\left(\mathrm{dd}, J_{\mathrm{H}-\mathrm{H}}=7.3\right.$ and $\left.1.4 \mathrm{~Hz}, 3 \mathrm{H}, \mathrm{CH}_{3} \mathrm{CHCHCO}\right), 0.92\left(\mathrm{dd}, J_{\mathrm{P}-\mathrm{H}}=6.1\right.$ and $1.8 \mathrm{~Hz}, 3 \mathrm{H}, \mathrm{Ir}-$ $\left.\mathrm{CH}_{3}\right) .{ }^{31} \mathrm{P}\left\{{ }^{1} \mathrm{H}\right\} \mathrm{NMR}\left(\mathrm{CD}_{2} \mathrm{Cl}_{2}\right)$ at $25{ }^{\circ} \mathrm{C}:(34 \%): \square 31.9\left(\mathrm{~d}, J_{\mathrm{P}-\mathrm{P}}=4.7 \mathrm{~Hz}, J_{\mathrm{P}-\mathrm{C}}=123\right.$ $\mathrm{Hz}, 1 \mathrm{P}$, trans to $\mathrm{CO}), 15.9\left(\mathrm{~d}, J_{\mathrm{P}-\mathrm{P}}=4.7 \mathrm{~Hz}, 1 \mathrm{P}\right.$, cis to $\left.\mathrm{CO}\right)$.

Binding study of dimethyl maleate and $\left[\operatorname{IrMe}(\mathrm{CO})(\mathrm{dppe})(\mathrm{DIB})_{2}\right][\mathrm{BARF}]_{2}$. The same procedure was followed as described above for binding study of N-crotonyl-2oxazolidinone and $\left[\mathrm{IrMe}(\mathrm{CO})(\mathrm{dppe})(\mathrm{DIB})_{2}\right][\mathrm{BARF}]_{2}$. Dimethyl maleate $(0.79 \square \mathrm{L}$, $0.0063 \mathrm{mmol}),\left[\mathrm{IrMe}(\mathrm{CO})(\mathrm{dppe})(\mathrm{DIB})_{2}\right][\mathrm{BARF}]_{2}(17.0 \mathrm{mg}, 0.0063 \mathrm{mmol})$, and $0.60 \mathrm{~mL}$ $\mathrm{CD}_{2} \mathrm{Cl}_{2}$ were used. ${ }^{1} \mathrm{H} \mathrm{NMR}\left(\mathrm{CD}_{2} \mathrm{Cl}_{2}\right)$ at $25^{\circ} \mathrm{C}$ : $\square 6.86-7.82$ (overlapping, $46 \mathrm{H}$, phenyl, BARF, olefinic), $4.12\left(\mathrm{~s}, 3 \mathrm{H}, \mathrm{COOCH}_{3}\right), 3.74\left(\mathrm{~s}, 3 \mathrm{H}, \mathrm{COOCH}_{3}\right), 3.0-3.50$ (overlapping $\left.\mathrm{m}, 4 \mathrm{H}, \mathrm{PCH} \mathrm{CH}_{2} \mathrm{P}\right), 0.92\left(\mathrm{dd}, J_{\mathrm{P}-\mathrm{H}}=5.7\right.$ and $\left.2.0 \mathrm{~Hz}, 3 \mathrm{H}, \mathrm{Ir}-\mathrm{CH}_{3}\right) .{ }^{1} \mathrm{P}\left\{{ }^{1} \mathrm{H}\right\} \mathrm{NMR}$ $\left(\mathrm{CD}_{2} \mathrm{Cl}_{2}\right)$ at $25^{\circ} \mathrm{C}: \square 33.6\left(\mathrm{~d}, J_{\mathrm{P}-\mathrm{P}}=4.7 \mathrm{~Hz}, 1 \mathrm{P}\right.$, trans to $\left.\mathrm{CO}\right), 17.2\left(\mathrm{~d}, J_{\mathrm{P}-\mathrm{P}}=4.7 \mathrm{~Hz}, 1 \mathrm{P}\right.$, cis to $\mathrm{CO})$.

General procedure for $\left[\mathrm{IrMe}(\mathrm{CO})(\mathrm{dppe})(\mathrm{DIB})_{2}\right][\mathrm{BARF}]_{2}$ catalyzed Nazarov cyclization. In a glovebox, an NMR tube was charged with 2-(benzo[1,3]dioxole-5carbonyl)-3-(2,4,6-trimethoxy-phenyl)-acrylic acid methyl ester (20.0 mg, $0.047 \mathrm{mmol})$ and $[\mathrm{IrMe}(\mathrm{CO})(\mathrm{dppe})(\mathrm{DIB})][\mathrm{BARF}]_{2}(2.5 \mathrm{mg}, 0.94 \square \mathrm{mol})$ and sealed with septum. The tube was cooled to $-78{ }^{\circ} \mathrm{C}, 0.60 \mathrm{~mL}$ of $\mathrm{CD}_{2} \mathrm{Cl}_{2}$ added and quickly transferred to precooled NMR spectrometer. The progress of the reaction was followed by ${ }^{1} \mathrm{H}$ NMR while warming to room temperature. ${ }^{1} \mathrm{H}$ NMR shows complete consumption of starting material in less than $20 \mathrm{~min}$. The reaction was purified by column chromatography on silica gel eluting with 50\% EtOAc/hexanes to give the cyclized product in $>99 \% .{ }^{1} \mathrm{H}$ $\operatorname{NMR}\left(\mathrm{CDCl}_{3}\right)$ at $25^{\circ} \mathrm{C}: \square 8.15(\mathrm{~s}, 1 \mathrm{H}), 7.48(\mathrm{q}, J=9.8 \mathrm{~Hz}, 1 \mathrm{H}), 7.44(\mathrm{~d}, J=1.6 \mathrm{~Hz}$, 
$1 \mathrm{H}), 6.79(\mathrm{~d}, J=8.1 \mathrm{~Hz}, 1 \mathrm{H}), 6.03(\mathrm{~s}, 2 \mathrm{H}), 5.96(\mathrm{~s}, 2 \mathrm{H}), 3.79(\mathrm{~s}, 3 \mathrm{H}), 3.72(\mathrm{~s}, 3 \mathrm{H}), 3.53$

(s, $6 \mathrm{H}) .{ }^{13} \mathrm{C}\left\{{ }^{1} \mathrm{H}\right\} \operatorname{NMR}\left(\mathrm{CDCl}_{3}\right)$ at $25^{\circ} \mathrm{C}: \quad \square 191.8,167.2,163.4,159.6,151.0,147.6$,

135.5, 132.5, 128.0, 125.1, 108.2, 107.5, 105.0, 101.5, 90.7, 55.2, 54.6, 52.0.

Kinetics study. Stock solutions of the catalyst and the appropriate substrate were

prepared in $\mathrm{CD}_{2} \mathrm{Cl}_{2}$. Calculated amounts of the catalyst solution and $\mathrm{CD}_{2} \mathrm{Cl}_{2}$ (to make up the required concentration) were transferred to NMR tube using Hamilton syringes and sealed with septum. The NMR tube was evacuated and backfilled three times with $\mathrm{N}_{2}$ at $-78{ }^{\circ} \mathrm{C}$ on a Schlenk-line. The required amount of pre-cooled $\left(-78^{\circ} \mathrm{C}\right)$ substrate, $17-50$ fold molar excess, was added and quickly transferred to pre-cooled NMR spectrometer. The ${ }^{1} \mathrm{H}$ NMR spectrum was recorded at $-60{ }^{\circ} \mathrm{C}$ to insure that no reaction has started. It was then brought to the desired temperature and the ensuing reaction was monitored by ${ }^{1} \mathrm{H}$ NMR spectroscopy. Each kinetics experiment was repeated two times. 


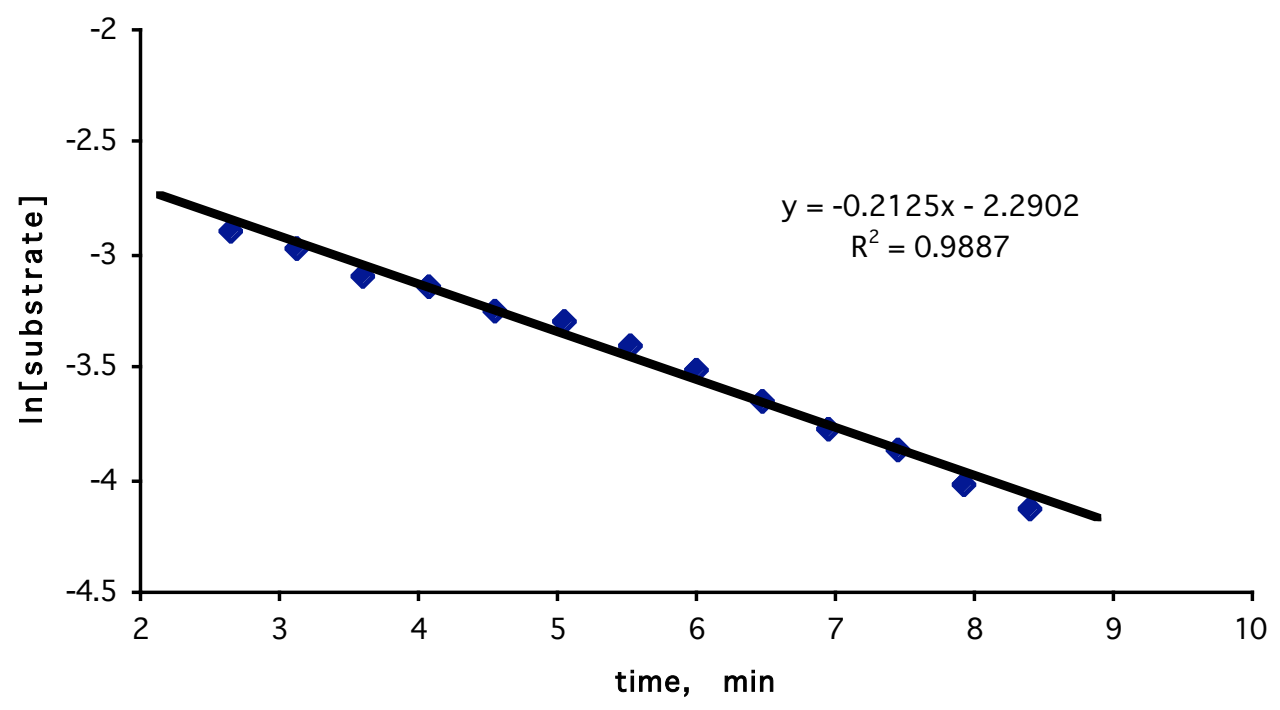

Figure 1. Plot of ln [substrate] versus time for Nazarov substrate 2-(benzo[1,3]dioxole5-carbonyl)-3-(2,4,6-trimethoxy-phenyl)-acrylic acid methyl ester and catalyst 1 in $\mathrm{CD}_{2} \mathrm{Cl}_{2}$ at $15{ }^{\circ} \mathrm{C}$. 


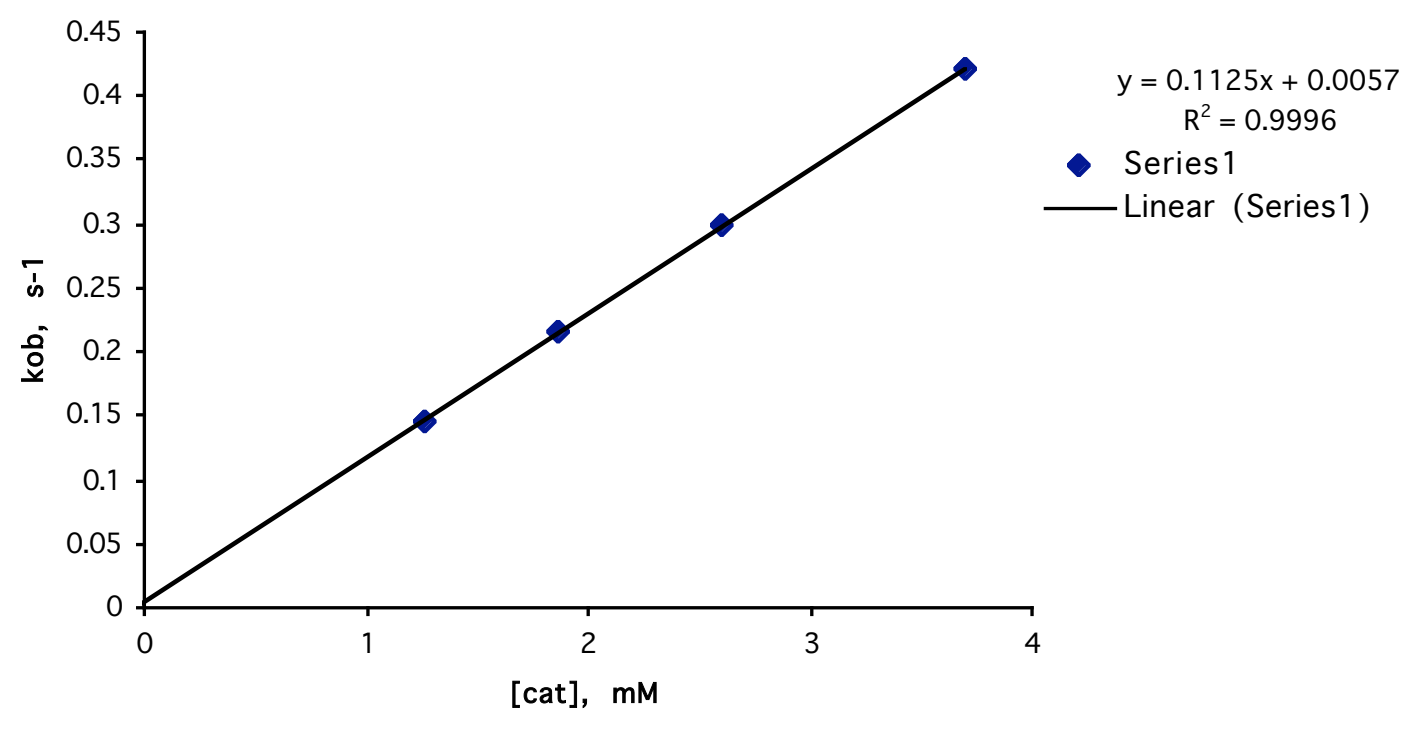

Figure 2. Plot of $\mathrm{k}_{\mathrm{ob}}$ versus [cat] for Nazarov substrate 2-(benzo[1,3]dioxole-5-

carbonyl)-3-(2,4,6-trimethoxy-phenyl)-acrylic acid methyl ester and catalyst $\mathbf{1}$ in $\mathrm{CD}_{2} \mathrm{Cl}_{2}$ at $15{ }^{\circ} \mathrm{C}$. 


\section{References}

(1) Albietz, P. J.; Cleary, B. P.; Paw, W.; Eisenberg, R. J. Am. Chem. Soc. 2001, 123, 12091-12092.

(2) Albietz, P. J.; Cleary, B. P.; Paw, W.; Eisenberg, R. Inorg. Chem. 2002, 41, 2095 2108.

(3) Brookhart, M.; Grant, B.; Volpe, J., A. F. Organometallics 1992, 11, 3920-3922.

(4) Jaquith, J. B.; Levy, C. J.; Bondar, G. V.; Wang, S.; Collins, S. Organometallics 1998, 17, 914-925.

(5) He, W.; Sun, X.; Frontier Alison, J. J. Am. Chem. Soc. 2003, 125, 14278-14279.

(6) Pangborn, A. B.; Giardello, M. A.; Grubbs, R. H.; Rosen, R. K.; Timmers, F. J. Organometallics 1996, 15, 1518-1520. 